\title{
Cauchy Problems with State-Dependent Time Evolution
}

\author{
A. Grabosch ${ }^{* \dagger}$ and H.J.A.M. Heimmans ${ }^{\dagger \dagger}$ \\ ${ }^{\dagger}$ Department of Mathematics, University of Tübingen, Auf der Morgenstelle 10 , \\ 7400-Tübingen, Federal Republic of Germany \\ t† Centre for Mathematics and Computer Science, \\ P.O. Box 4079, 1009 AB Amsterdam, The Netherlands
}

Received February 28, 1989

Revised August 23, 1989

\begin{abstract}
We consider a class of quasilinear Cauchy problems which frequently arise in the context of structured population dynamics and which have in common that they can be reduced to a semilinear problem by a time-scale argument. We prove existence and uniqueness of solutions, study positivity and regularity properties, and prove the principle of linearized stability. These abstract results are applied to a model describing the dynamics of a sizestructured cell population whose individuals are subject to a nonlinear growth law.
\end{abstract}

Key words: quasilinear Cauchy problem, state-dependent time evolution, strongly continuous semigroup, semilinear Cauchy problem, variation-of-constants formula, global solution, nonlinear semigroup, positivity, linearized stability, structured population dynamics, size-dependent cell growth and division

\section{Statement of the Problem and Motivation}

Many models of structured population dynamics have in common that individuals are assumed to interact through their environment: see Metz, Diekmann [12]. One may think, for example of a situation where individuals (of one or more species) all consume from a common resource pool, and where the per capita growth-, reproduction-, and death-rate depend on this consumption. The number of individuals affects the food availability which again affects the population dynamical processes on the individual level. The case where individuals produce some chemical substance which inhibits their reproduction may serve as another example.

Often such models can be properly described by an abstract Cauchy problem of the form

$$
\begin{aligned}
& \frac{d}{d t} u(t)=A(E(t)) u(t), \\
& \frac{d}{d t} E(t)=f(u(t), E(t)) .
\end{aligned}
$$

Here $E(t)$ describes the environment (a scalar-or vector-valued function) and $u(t)$ describes the population distribution at time $t$. For every fixed $t, u(t)$ can be

* This work was done during a visit to the Centre of Mathematics and Computer Science, Amsterdam, in the academic year $1987 / 1988$. The stay was supported by the Stichting Mathematisch Centrum. I cordially thank my hosts for making this stay possible and very much enjoyable. Especially, I thank all the members of the "Applied Mathematics Department" for their great hospitality and for many valuable discussions. 
considered as an element of some Banach space $X$. For every possible environment $E$, the (partial differential) operator $A(E)$ is the infinitesimal generator of a strongly continuous semigroup of the linear operators on $X$. Actually, the operator $A(E)$ is obtained from a careful bookkeeping of all processes on the individual level. Finally, $f: X \times \boldsymbol{R}^{n} \rightarrow \boldsymbol{R}^{n}$ is some nonlinear function describing the interaction of the individuals with the environment. Usually, the dependence of $f$ on $u$ is through some linear functional. From the interpretation it follows that $E$ should be positive and that $u$ should take values in a positive cone $X_{+}$.

Nonlinear growth mechanisms have been considered before by Diekmann, Lauwerier, Aldenberg and Metz [6], Heijmans [8], Kooijman and Metz [10], Murphy [13] and Tucker and Zimmermann [17]. The linear version of the model discussed here has been treated using semigroup methods by Diekmann, Heijmans and Thieme [5], Diekmann and Metz [12, Chapter II], and Greiner and Nagel [7].

As a particular example of (1.1) we mention the following model. Suppose we have a size-structured population whose individuals reproduce by division into two equal halves. Suppose moreover that the cells produce some enzyme which has a restraining effect on the growth rate of individual cells and in this manner controls the size of the whole population. This situation can be described by the following system of equations:

$$
\begin{aligned}
\frac{\partial}{\partial t} u(t, s) & +\frac{\partial}{\partial s}(\widehat{\gamma}(E(t)) g(s) u(t, s)) \\
& =-\mu(s) u(t, s)-b(s) u(t, s)+4 b(2 s) u(t, 2 s) \\
\frac{d}{d t} E(t) & =-\sigma E(t)+\int h(s) u(t, s) d s .
\end{aligned}
$$

Here $s$ is size, $u(t, \cdot)$ denotes the population density, $E(t)$ is the enzyme concentration at time $t, \widehat{\gamma}(E) g(s)$ is the individual growth rate of a cell with size $s$ at enzyme concentration $E, \mu(s)$ is the death rate, and $b(s)$ is the division rate. Furthermore $h(s)$ denotes the production rate of the enzyme by a cell with size $s$, and $\sigma$ the desintegration rate of the enzyme. In Section 6 the model is discussed in more detail. Here we formulate a rather general class of abstract Cauchy problems in which (1.2) (but not (1.1)) is contained, and which is the main subject of this paper.

Let $X$ be an arbitrary Banach space, $A_{0}$ the infinitesimal generator of a $C_{0}$ semigroup of linear operators $\left\{T_{0}(t), t \geq 0\right\}$ on $X$, and $F: X \rightarrow X$ a linear or nonlinear continuous operator. Finally, let $\gamma: X \rightarrow \boldsymbol{R}_{+}$be a continuous function. We consider the abstract Cauchy problem

$$
\begin{aligned}
& \frac{d}{d t} u(t)=\gamma(u(t)) A_{0} u(t)+F(u(t)) \quad \text { for } t \geq 0, \\
& u(0)=x
\end{aligned}
$$

where $x \in X$.

This type of Cauchy'problems is typical for many structured population models. Quite often $\gamma(u)$ is of the special form

$$
\gamma(u)=\widehat{\gamma}\left(L_{1}(u), L_{2}(u), \ldots, L_{m}(u)\right),
$$


where $L_{1}, L_{2}, \ldots, L_{m}$ are linear functionals on $X$ and $\hat{\gamma}$ is a real function of $m$ variables. In Section 6 it is shown that this also holds for the model in (1.2): there we also give explicit expressions of $\gamma, A_{0}$, and $F$ for this model.

Our interest in $\left(\mathrm{P}_{t}\right)$ originates from the study of a structured model of the blood cell production system. In the future we intend to consider a version of $\left(\mathrm{P}_{t}\right)$ involving the duality framework of Clément et al. [2], [3] and to apply it to our model of the blood cell production system.

In this paper we will investigate the abstract Cauchy problem $\left(\mathrm{P}_{t}\right)$. The key idea is to reduce this quasilinear problem to a semilinear problem by a time-scale argument. A general approach towards quasilinear equations is given by Kato [9] (see also Pazy [15]). For our situation the direct approach presented here is more efficient and leads to a better understanding of the dynamics. In Section 2 we will discuss the time-scaling transformation which reduces $\left(\mathrm{P}_{t}\right)$ to a semilinear equation. This transformation is extensively used in Section 3 where we examine existence, uniqueness and regularity of solutions, and in Section 4, where we examine positivity and boundedness properties. We shall deal with the principle of linearized (in) stability in Section 5. Finally, in Section 6, we shall work out example (1.2) in some detail.

\section{The Time-Scaling Transformation}

In this section we shall define and examine a mapping which transforms the quasilinear Cauchy problem $\left(\mathrm{P}_{t}\right)$ into a semilinear one. First we introduce some notation and terminology. Let $X$ be a Banach space and let $\gamma$ be a function from $X$ to $\boldsymbol{R}_{+}$satisfying the following assumption.

AsSUMPtion $2.1 \quad \gamma$ is a continuous, strictly positive, and locally bounded function, i.e., $\gamma$ is bounded on bounded subsets of $X$.

For every continuous function $u:\left[0, t_{0}\right] \rightarrow X$ we define

$$
\tau_{u}(t):=\int_{0}^{t} \gamma(u(s)) d s, \quad t \in\left[0, t_{0}\right] .
$$

Analogously, if $v:\left[0, \tau_{0}\right] \rightarrow X$ is a continuous function, we define

$$
t_{v}(\tau):=\int_{0}^{\tau}[\gamma(v(\sigma))]^{-1} d \sigma, \quad \tau \in\left[0, \tau_{0}\right] .
$$

Proposition 2.2. To every $t_{0} \geq 0$ and $u \in C\left(\left[0, t_{0}\right] ; X\right)$ there corresponds a unique $\tau_{0} \geq 0$ and a unique $v \in C\left(\left[0, \tau_{0}\right] ; X\right)$ such that the following relations hold:

$$
\begin{aligned}
& \tau_{0}=\tau_{u}\left(t_{0}\right) \\
& t_{0}=t_{v}\left(\tau_{0}\right) \\
& t_{v}\left(\tau_{u}(t)\right)=t \quad 0 \leq t \leq t_{0}
\end{aligned}
$$




$$
\begin{array}{lc}
\tau_{u}\left(t_{v}(\tau)\right)=\tau, & 0 \leq \tau \leq \tau_{0} \\
v\left(\tau_{u}(t)\right)=u(t), & 0 \leq t \leq t_{0} \\
u\left(t_{v}(\tau)\right)=v(\tau), & 0 \leq \tau \leq \tau_{0} .
\end{array}
$$

Conversely, to every $\tau_{0} \geq 0$ and $v \in C\left(\left[0, \tau_{0}\right] ; X\right)$ there corresponds a unique $t_{0} \geq 0$ and a unique $u \in C\left(\left[0, t_{0}\right] ; X\right)$ such that the above relations hold.

If $u \in C\left(\left[0, t_{0}\right] ; X\right)$ then we denote by $v=v[u]$ the element of $C\left(\left[0, \tau_{0}\right] ; X\right)$ defined by (2.7), where $\tau_{0}$ is given by (2.3). Similarly, $u=u[v]$ is defined. It follows immediately that

$$
u[v[u]]=u \quad \text { and } \quad v[u[v]\}=v .
$$

Our next concern is the semigroup property. Suppose that for every $x \in X$ we have a function $u(\cdot ; x) \in C\left(\left[0, t_{0}(x)\right) ; X\right)$, and let $v(\cdot, x)=v[u(\cdot ; x)]$ be the corresponding function in $C\left(\left[0, \tau_{0}(x)\right) ; X\right)$, where $\tau_{0}(x)=\tau_{u(\cdot ; x)}\left(t_{0}(x)\right)$. We say that $u(\cdot ; x)$ has the semigroup property if $t_{0}(u(s ; x)) \geq t_{0}(x)-s$ for $s \leq t_{0}(x)$, and

$$
u(t+s ; x)=u(t ; u(s ; x)) \text { if } t+s \leq t_{0}(x) .
$$

Proposition 2.3. Let $u(\cdot ; x)$ and $v(\cdot ; x)$ be as above. Then $u$ has the semigroup property if and only if $v$ has the semigroup property.

Proof. Suppose that $v$ has the semigroup property. We show that $u$ has the semigroup property as well. We use the following notation. For $x \in X$ we define

$$
t(\tau ; x):=t_{v(\cdot ; x)}(\tau)=\int_{0}^{\tau}[\gamma(v(\sigma ; x))]^{-1} d \sigma
$$

and let $\tau(\cdot ; x)$ be its inverse. Thus $u(\cdot ; x)$ is given by

$$
u(t ; x):=v(\tau(t ; x) ; x), \quad \text { for } t \in\left[0, t_{0}(x)\right) .
$$

We have to show that

$$
u(t+s ; x)=u(t ; u(s ; x))
$$

for $s, t \geq 0$ with $t+s<t_{0}(x)$. From (2.9) we easily deduce that

$$
t(\tau ; v(\sigma ; x))+t(\sigma ; x)=t(\tau+\sigma ; x) .
$$

Substitution of $\tau=\tau_{0}(u(s ; x))$ and $\sigma=\tau(s ; x)$ in $(2.11)$ and using that $\tau_{0}(v(\sigma ; x))+$ $\sigma \geq \tau_{0}(x)$ yields that

$$
\begin{aligned}
t_{0}(u(s ; x)) & =t\left(\tau_{0}(u(s ; x)) ; u(s ; x)\right) \\
& =t\left(\tau_{0}(u(s ; x)) ; v(\sigma ; x)\right) \\
& =t\left(\tau_{0}(v(\sigma ; x))+\sigma ; x\right)-t(\sigma ; x) \\
& \geq t\left(\tau_{0}(x) ; x\right)-s=t_{0}(x)-s,
\end{aligned}
$$


whence the first part of the conjecture follows. To prove that $u(t+s ; x)=u(t ; u(s ; x))$ if $t+s<t_{0}(x)$, we substitute $\tau=\tau(t ; v(\tau(s ; x) ; x))$ and $\sigma=\tau(s ; x)$ in $(2.11)$, to obtain

$$
t+s=t(\tau(t ; v(\tau(s ; x) ; x))+\tau(s ; x) ; x)
$$

or equivalently,

$$
\tau(t+s ; x)=\tau(t ; v(\tau(s ; x) ; x))+\tau(s ; x) .
$$

Thus, using that $u(t ; x)+v(\tau(t ; x) ; x)$ and the fact that $v$ satisfies the semigroup property, we find that

$$
\begin{aligned}
u(t+s ; x) & =v(\tau(t+s ; x) ; x) \\
& =v(\tau(t ; v(\tau(s ; x) ; x))+\tau(s ; x) ; x) \\
& =v(\tau(t ; v(\tau(s ; x) ; x)) ; v(\tau(s ; x) ; x)) \\
& =v(\tau(t ; u(s ; x)) ; u(s ; x)) \\
& =u(t ; u(s ; x)),
\end{aligned}
$$

for $t+s<t_{0}(x)$.

To solve the quasilinear Cauchy problem $\left(\mathrm{P}_{t}\right)$ we apply the transformation $v(\tau):=u\left(\tau_{u}^{-1}(\tau)\right)$, where $\tau_{u}^{-1}$ is the inverse function of $\tau_{u}$, which is defined in (2.1). This transformation yields the following semilinear Cauchy problem for $v$ :

$$
\begin{aligned}
& \frac{d}{d \tau} v(\tau)=A_{0} v(\tau)+B(v(\tau)) \text { for } \tau \geq 0 \\
& v(0)=x
\end{aligned}
$$

where the (nonlinear) operator $B$ given by

$$
B(v):=F(v) / \gamma(v)
$$

maps $X$ into itself. We shall explain the relation between the two problems and their respective solutions in more detail. The variation-of-constants formulas corresponding to $\left(\mathrm{P}_{t}\right)$ and $\left(\mathrm{P}_{\tau}\right)$ respectively look as follows:

$$
\begin{aligned}
& u(t)=T_{0}\left(\tau_{u}(t)\right) x+\int_{0}^{t} T_{0}\left(\tau_{u}(t)-\tau_{u}(s)\right) F(u(s)) d s \\
& v(\tau)=T_{0}(\tau) x+\int_{0}^{\tau} T_{0}(\tau-\sigma) B(v(\sigma)) d \sigma
\end{aligned}
$$

Definition 2.4. We call $u:\left[0, t_{0}\right] \rightarrow X$ a (local) classical solution of $\left(\mathrm{P}_{t}\right)$ if $u$ is continuously differentiable, $u(t) \in D\left(A_{0}\right)$ for all $t \in\left[0, t_{0}\right]$, and $u$ satisfies equation $\left(\mathrm{P}_{t}\right)$. Analogously, (local) classical solutions of $\left(\mathrm{P}_{\tau}\right)$ are defined. A continuous function $u:\left[0, t_{0}\right] \rightarrow X$ is called a (local) mild solution of $\left(\mathrm{P}_{t}\right)$ if $u$ satisfies $\left(\mathrm{VOC}_{t}\right)$. Similarly, (local) mild solutions of $\left(\mathrm{P}_{\tau}\right)$ are defined. 
Theorem 2.5. Let $u \in C\left(\left[0, t_{0}\right] ; X\right)$ and let $v=v[u]$.

(a) $u$ is a (local) classical solution of $\left(\mathrm{P}_{t}\right)$ if and only if $v$ is a (local) classical solution of $\left(\mathrm{P}_{\tau}\right)$.

(b) $u$ is a (local) mild solution of $\left(\mathrm{P}_{t}\right)$ if and only if $v$ is a (local) mild solution of $\left(\mathrm{P}_{\tau}\right)$.

Proof. (a): Let $u$ be a classical solution of $\left(\mathrm{P}_{t}\right)$. Thus $u \in C^{1}\left(\left[0, t_{0}\right] ; X\right), u(t) \in$ $D\left(A_{0}\right)$ for $0 \leq t \leq t_{0}$, and $u$ satisfies $\left(\mathrm{P}_{t}\right)$. Let $v=v[u]$, then $v \in C^{1}\left(\left[0, \tau_{0}\right] ; X\right)$ and $v(\tau) \in D\left(A_{0}\right)$ for $0 \leq \tau \leq \tau_{0}$. Differentiation of the identity $u(t)=v\left(\tau_{u}(t)\right)$ with respect to $t$ yields

$$
\frac{d v}{d \tau}\left(\tau_{u}(t)\right)=A_{0} v\left(\tau_{u}(t)\right)+B\left(v\left(\tau_{u}(t)\right)\right.
$$

Substituting $t=t_{v}(\tau)$ and using Proposition 2.2 yields

$$
\frac{d v}{d \tau}(\tau)=A_{0} v(\tau)+B(v(\tau))
$$

Thus $v$ is a classical solution of $\left(\mathrm{P}_{\tau}\right)$.

The proof of the "if"-statement proceeds along the same lines.

(b): Let $u$ be a solution of $\left(\mathrm{VOC}_{t}\right)$. By substitution of $t=t_{v}(\tau)$ and $s=t_{v}(\sigma)$ one easily finds that $v=v[u]$ is a solution of $\left(\mathrm{VOC}_{\tau}\right)$. The converse is proved similarly.

Theorem 2.5 provides a rigorous justification of the intuitive idea that solving the quasilinear equation $\left(\mathrm{P}_{t}\right)$ amounts to solving the semilinear equation $\left(\mathrm{P}_{\tau}\right), \mathrm{a}$ problem which, as we shall see in the next section, is well understood.

\section{Solving the Quasilinear Equation}

In this section we address the question of existence, uniqueness, and regularity of solutions of the quasilinear equation $\left(\mathrm{P}_{t}\right)$. At one hand, the semilinear problem can be considered as a special case of the quasilinear problem, namely with $\gamma \equiv 1$. On the other hand, the results of the previous section (in particular, Theorem 2.5) tell us that the two problems are in fact equivalent. We exploit this latter fact in this and the following section. Most of the results here will be stated for the quasilinear problem. In the proofs, however, thanks to the time-scaling transformation, we may restrict to the semilinear case. There is one exception, namely where we relate the semigroup solution of the linear Cauchy problem, which we get by linearizing around an equilibrium, to the original nonlinear semigroup: in the semilinear case the first is the Fréchet derivative of the latter at the equilibrium, but in the quasilinear case this needs not be the case. We refer to Section 5 for more details.

Throughout the remainder of this paper we make Assumption 2.1 along with

Assumption 3.1. The operator $B$ defined by

$$
B(u):=F(u) / \gamma(u)
$$


is locally Lipschitz continuous.

The latter means that for $r \geq 0$ there exists a constant $C(r) \geq 0$ such that

$$
\|B(x)-B(y)\| \leq C(r)\|x-y\|
$$

for all $x, y \in X$ with $\|x\| \leq r,\|y\| \leq r$.

First we will study the existence of continuous solutions of $\left(\mathrm{VOC}_{t}\right)$. In Theorems 3.3 and 3.4 we will state some regularity results for these solutions. For related results see also Clément et al. [3]. It is well known that local Lipschitz continuity of $B$ implies the existence of solutions of $\left(\mathrm{VOC}_{\tau}\right)$ on a maximal interval. This fact is exploited in the following existence theorem.

Theorem 3.2. For every $x \in X$ there exists a maximal $t_{\max }(x)>0$ such that $\left(\mathrm{VOC}_{t}\right)$ has a unique continuous solution $u(\cdot ; x)$ on $\left[0, t_{\max }(x)\right)$ which has the semigroup property. If $t_{\max }(x)<\infty$, then $\lim _{t \uparrow t_{\max }}\|u(t ; x)\|=\infty$.

Proof. Since $B$ is locally Lipschitz continuous there exists a continuous solution $v(\tau ; x)$ of $\left(\mathrm{VOC}_{\tau}\right)$ on $\left[0, \tau_{\max }\right)$ for every $x \in X$. Furthermore, this solution $v$ has the semigroup property, that is,

$$
v(\tau ; v(\sigma ; x))=v(\tau+\sigma ; x), \quad \text { for } \tau, \sigma>0 \text { with } \quad \tau+\sigma<\tau_{\max }
$$

Using Theorem 2.5(b) we can conclude that, for every $x \in X$, there exists a continuous solution $u(t ; x)$ of $\left(\mathrm{VOC}_{t}\right)$ on some interval $\left[0, t_{\max }\right)$. Here

$$
t_{\max }=t_{\max }(x):=\lim _{\tau \uparrow \tau_{\max }} t_{v}(\tau)=\int_{0}^{\tau_{\max }}[\gamma(v(\sigma ; x))]^{-1} d \sigma .
$$

Proposition 2.3 tells us that $u$ inherits the semigroup property from $v$. Suppose $t_{\max }<\infty$ and that $\|u(t ; x)\| \leq C$ for $t<t_{\max }$. Then $\tau_{\max }=\int_{0}^{t_{\max }} \gamma(u(s ; x)) d s<\infty$ and therefore $\|v(\tau ; x)\| \rightarrow \infty$ as $\tau \uparrow \tau_{\max }$. But $\|v(\tau ; x)\|=\|u(t(\tau ; x) ; x)\| \leq C$ for $\tau \in\left[0, \tau_{\max }\right)$, a contradiction. From this the result follows.

In order to find solutions of $\left(\mathrm{P}_{t}\right)$ in a "strong" sense, one has to study the regularity properties of solutions of $\left(\mathrm{VOC}_{t}\right)$. A first result generalizing a theorem of Ball [1] shows that continuous solutions of $\left(\mathrm{VOC}_{t}\right)$ are indeed "weak solutions" of $\left(P_{t}\right)$ in the following sense:

TheOREM 3.3. Let $u(\cdot):=u(\cdot ; x)$ be a continuous solution of $\left(\mathrm{VOC}_{t}\right)$ on $\left[0, t_{0}\right)$ with initial value $x \in X$. Then $\left\langle u(\cdot), x^{*}\right\rangle$ is continuously differentiable for every $x^{*} \in D\left(A_{0}^{*}\right)$ and

$$
\frac{d}{d t}\left\langle u(t), x^{*}\right\rangle=\gamma(u(t))\left\langle u(t), A_{0}^{*} x^{*}\right\rangle+\left\langle F(u(t)), x^{*}\right\rangle, \quad \text { for all } t \in\left[0, t_{0}\right)
$$


Proof. Let $v=v[u]$ be the corresponding solution of $\left(\mathrm{VOC}_{\tau}\right)$ on $\left[0, \tau_{0}\right)$. From Ball's result we know that $\left\langle v(\cdot), x^{*}\right\rangle$ is continuously differentiable, and that

$$
\frac{d}{d \tau}\left\langle v(\tau), x^{*}\right\rangle=\left\langle v(\tau), A_{0}^{*} x^{*}\right\rangle+\left\langle B(v(\tau)), x^{*}\right\rangle, \quad \text { for all } \tau \in\left[0, \tau_{0}\right) .
$$

Using (2.10) the corresponding result for $u$ follows easily.

So continuous solutions of $\left(\mathrm{VOC}_{t}\right)$ are weak solutions of $\left(\mathrm{P}_{t}\right)$. We now look for conditions under which the continuous solutions of $\left(\mathrm{VOC}_{t}\right)$ are classical solutions of $\left(\mathrm{P}_{t}\right)$.

Theorem 3.4. Assume that $B$ is continuously Fréchet differentiable. Then any continuous solution $u(\cdot ; x)$ of $\left(\mathrm{VOC}_{t}\right)$ is a classical solution of $\left(\mathrm{P}_{t}\right)$ if $x \in$ $D\left(A_{0}\right)$.

Proof. It follows from Pazy [5, Chapter 6, Theorem 1.5] that a continuous solution $v$ of $\left(\mathrm{VOC}_{\tau}\right)$ is a classical solution of $\left(\mathrm{P}_{\tau}\right)$ for initial data $x$ in $D\left(A_{0}\right)$. Now the corresponding result for $u$ follows immediately if one uses (2.10).

The main assertions so far can be summarized as follows. Theorem 3.2 assures the existence of local solutions $u(\cdot ; x)$ of $\left(\mathrm{VOC}_{t}\right)$. If this solution stays bounded on every open interval $\left[0, t_{0}\right)$ then, again by Theorem 3.2 , the integral equation $\left(\mathrm{VOC}_{t}\right)$ possesses a global solution, or in other words $t_{\max }(x)=\infty$. If this holds for every $x \in X$, then we can define a family of operators $\{T(t), t \geq 0\}$ by

$$
T(t) x:=u(t ; x) \text { for } t \in[0, \infty) \text { and } x \in X .
$$

The operators $T(t)$ form a strongly continuous nonlinear semigroup on $X$. Actually, it is remarkable that the thus obtained semigroup is usually not quasi-dissipative as in the setting of Crandall-Liggett type theorems.

We conclude this section with an example (based upon ideas of Mats Gyllenberg) which illustrates that it is not merely sufficient to assume that $\gamma$ is nonnegative, but that strict positivity is a requirement which cannot be dispensed with.

Example 3.5. Consider the quasilinear Cauchy problem

$$
\frac{d}{d t} u(t)=\gamma(u(t)) A_{0} u(t), \quad u(0)=x,
$$

where $A_{0}$ is the generator of a $C_{0}$-semigroup $\left\{T_{0}(t), t \geq 0\right\}$ on $X$. The integral equation corresponding to this problem is:

$$
u(t ; x)=T_{0}\left(\int_{0}^{t} \gamma(u(s ; x)) d s\right) x .
$$

Indeed, for $x \in D\left(A_{0}\right)$, every continuous solution of (3.4) is automatically continuously differentiable and satisfies (3.3). Nevertheless solutions of (3.4) are not always unique as we now show. 
Let $X$ be the Banach space

$$
X:=\left\{x \in C\left(\boldsymbol{R}_{+}\right) \mid \theta \mapsto e^{-\theta} x(\theta) \text { is bounded and uniformly continuous }\right\}
$$

with norm $\|x\|:=\sup _{\theta \geq 0} e^{-\theta}|x(\theta)|$. Let $\left\{T_{0}(t), t \geq 0\right\}$ be the $C_{0}$-semigroup of translations on $X$, or, to be specific,

$$
\left(T_{0}(t) x\right)(\theta):=x(t+\theta), \quad t, \theta \geq 0
$$

Let $\gamma(u):=|u(0)|$ and let $x \in X$ be given by $x(\theta):=2 \sqrt{\theta}$. It is easy to check that for every $c \in \boldsymbol{R}_{+}$

$$
u_{c}(t ; x)(\theta):= \begin{cases}2 \sqrt{\theta} & \theta \geq 0, t<c, \\ 2 \sqrt{\theta+(t-c)^{2}}, & \theta \geq 0, t \geq c,\end{cases}
$$

is a solution of integral equation (3.4). Using the definition of $T_{0}(t)$ the integral equation (3.4) amounts to

$$
u(t ; x)(\theta)=x\left(\theta+\int_{0}^{t}|u(s ; x)(0)| d s\right) .
$$

Note that $x$ is an equilibrium of the system (3.4) even though $x \notin D\left(A_{0}\right)$. In fact, every initial condition $x$ with $x(0)=0$ is an equilibrium. By substitution of $\theta=0$, equation (3.5) can be reduced to the scalar integral equation

$$
u(t ; x)(0)=x\left(\int_{0}^{t} u(s ; x)(0) d s\right)
$$

as long as we consider only nonnegative initial data. Moreover, for $\psi(t):=$ $\int_{0}^{t} u(s ; x)(0) d s$ this equation reduces to the ordinary differential equation:

$$
\psi^{\prime}(t)=x(\psi(t)), \quad \psi(0)=0
$$

Clearly, the nonuniqueness of solutions of this differential equation is a consequence of the fact that $x$ is not Lipschitz continuous.

\section{Positivity and Boundedness}

As motivated in the introduction we are interested in equations of form $\left(\mathrm{P}_{t}\right)$ which are derived from models for structured populations, and whose solutions describe the distribution of the individuals with respect to some structuring variable(s) such as (physiological) age and size. The only biologically relevant solutions $u(\cdot ; x)$ of $\left(\mathrm{P}_{t}\right)$ are those which are positive as long as we start with a positive initial distribution $x$. Thus we are interested in conditions, preferably on $A_{0}$ and $F$, which assure that solutions $u(\cdot ; x)$ of $\left(\mathrm{P}_{t}\right)$ are positive given that the initial data are positive. Whenever we speak of positivity, we assume that $X$ is a Banach lattice and 
we denote the positive cone by $X_{+}$(see Schaefer [16]). If $x$ is an element of $X_{+}$ then we write $x \geq 0$.

An easy consequence of the variation-of-constants formula $\left(\mathrm{VOC}_{t}\right)$ is the following

Proposition 4.1. Let $A_{0}$ be the generator of a linear positive $C_{0}$-semigroup $\left\{T_{0}(t), t \geq 0\right\}$ on $X$ and let $F$ map $X_{+}$into $X_{+}$. If $x \geq 0$ then $u(t ; x) \geq 0$ for all $t \in\left[0, t_{\max }(x)\right)$. Here $u(\cdot ; x)$ is the solution of $\left(\mathrm{VOC}_{t}\right)$ given by Theorem 3.2 .

In applications it seems too strong to assume that $F$ is positive. We want to allow some "local" nonpositivity. Thus we claim that the assertion of Proposition 4.1 remains valid under a weaker positivity assumption on $F$.

TheOREM 4.2. Let $A_{0}$ be the generator of a linear positive $C_{0}$-semigroup $\left\{T_{0}(t), t \geq 0\right\}$ and let $F$ satisfy the following "positive-off-diagonal" property

$$
\left\langle F(x), x^{*}\right\rangle \geq 0 \text { for all } x \geq 0, x^{*} \geq 0 \text { with }\left\langle x, x^{*}\right\rangle=0 .
$$

Then $x \geq 0$ implies $u(t ; x) \geq 0$ for all $t \in\left[0, t_{\max }(x)\right)$.

Theorem 4.2 can easily be proved for $\gamma \equiv 1$ and $F$ a linear bounded operator on $X$. Indeed by Proposition 4.1 we know that $A_{0}+F+\|F\| I$ generates a positive semigroup, since $F+\|F\| I \geq 0$ : see Nagel [14, Section C-II, Theorem 1.11]. Hence $A_{0}+F=A_{0}+F\|F\| I-\|F\| I$ generates a positive semigroup as well.

In the nonlinear case the analysis is somewhat more tedious. We will need some results concerning the geometry of the positive cone $X_{+}$. For $x \in X$ we denote by $x_{+}:=\sup \{x, 0\}, x_{-}:=\sup \{-x, 0\}$ and $|x|:=\sup \{x,-x\}$ the positive part, the negative part and the modulus of $x$, respectively. Furthermore let $\operatorname{dist}\left(x, X_{+}\right):=$ $\inf _{y \in X_{+}}\|x-y\|$ denote the distance function.

Lemma 4.3. Let $X$ be a Banach lattice. Then

(a) $x=x_{+}-x_{-}$and $|x|=x_{+}+x_{-}$for all $x \in X$;

(b) $x \mapsto x_{+}, x \mapsto x_{-}$and $x \mapsto|x|$ are continuous;

(c) $\|x-y\| \geq\left\|x_{-}\right\|-\left\|y_{-}\right\|$for all $x, y \in X$;

(d) $x \mapsto\left\|x_{-}\right\|$is convex and positive homogeneous, i.e. $\left\|\lambda x_{-}\right\|=\lambda\left\|x_{-}\right\|$for all $x \in X$ and $\lambda \geq 0$;

(e) $\left\|x_{-}\right\|=\operatorname{dist}\left(x, X_{+}\right)$for all $x \in X$;

(f) $\operatorname{dist}\left(x+y, X_{+}\right) \leq \operatorname{dist}\left(x, X_{+}\right)+\operatorname{dist}\left(y, X_{+}\right)$for all $x, y \in X$;

(g) $\quad\left\|[x+y]_{-}\right\| \leq\left\|x_{-}\right\|+\|y-\| \leq\left\|x_{-}\right\|+\|y\|$ for all $x, y \in X$;

(h) Let $T$ be a positive linear operator on $X$. Then $(T x)_{-} \leq T x_{-}$for all $x \in X$.

Proof. (a), (b): easy.

(c): For $z_{1}, z_{2} \in X$ we have $\left(z_{1}+z_{2}\right)_{-} \leq\left(z_{1}\right)_{-}+\left(z_{2}\right)_{-}$. Indeed let $z_{3}=z_{1}+z_{2}$. Since $\left(z_{i}\right)_{-}=\frac{1}{2}\left(\left|z_{i}\right|-z_{i}\right)(i=1,2,3)$ we have $\left(z_{3}\right)_{-} \leq \frac{1}{2}\left(\left|z_{1}\right|+\left|z_{2}\right|-z_{1}-z_{2}\right)=$ $\left(z_{1}\right)_{-}+\left(z_{2}\right)_{-}$. Let $x, y \in X$. Then $x_{-}=(x-y+y)_{-} \leq(x-y)_{-}+y_{-}$. For the norm we obtain $\left\|x_{-}\right\| \leq\left\|[x-y]_{-}\right\|+\left\|y_{-}\right\| \leq\|x-y\|+\left\|y_{-}\right\|$. Thus $\|x-y\| \geq\left\|x_{-}\right\|-\left\|y_{-}\right\|$ for all $x, y \in X$. 
(d): easy.

(e): Let $x, y \in X$. Then $\operatorname{dist}\left(x, X_{+}\right)=\inf _{y \in X_{+}}\|x-y\| \leq\left\|x-x_{+}\right\|=\left\|x_{-}\right\|$. For the converse estimate we conclude from (c) that $\|x-y\| \geq\left\|x_{-}\right\|-\left\|y_{-}\right\|=\left\|x_{-}\right\|$for all $y \in X_{+}$. Hence $\operatorname{dist}\left(x, X_{+}\right)=\inf _{y \in X_{+}}\|x-y\| \geq\left\|x_{-}\right\|$which proves assertion (e).

(f): Let $x, y \in X$. Then $\operatorname{dist}\left(x+y, X_{+}\right)=\inf _{z \in X_{+}}\|x+y-z\|$ $=\inf _{u \in X_{+}} \inf _{v \in X_{+}}\|x+y-u-v\| \leq \inf _{u \in X_{+}}\|x-u\|+\inf _{v \in X_{+}}\|y-v\|=\operatorname{dist}\left(x, X_{+}\right)+$ $\operatorname{dist}\left(y, X_{+}\right)$.

(g): easy.

(h): Let $0 \leq T \in \mathcal{L}(X)$ and $x \in X$. Then $(T x)_{-}=\frac{1}{2}(|T x|-T x) \leq \frac{1}{2}(T|x|-$ $T x)=T x_{-}$.

LEMMA 4.4 Let $x \geq 0$. Equivalent are:

(i) If $x^{*} \geq 0$ and $\left\langle x, x^{*}\right\rangle=0$, then $\left\langle F(x), x^{*}\right\rangle \geq 0$.

(ii) $\lim _{h \downarrow 0} \frac{1}{h} \operatorname{dist}\left(x+h F(x), X_{+}\right)=\lim _{h \downarrow 0} \frac{1}{h}\|[x+h F(x)]-\|=0$.

Proof. We can assume without loss of generality that $x \in \partial X_{+}$. Indeed for $x \in \operatorname{int}\left(X_{+}\right)$the conditions (i) and (ii) are trivially satisfied.

Consider $\Phi: X \rightarrow \mathbb{R}_{+}$given by $\Phi(x)=\operatorname{dist}\left(x, X_{+}\right)$. Lemma 4.3 gives us that $\Phi$ is a sublinear, continuous function. We thus can define the subdifferential $d \Phi(x)$ of $\Phi$ in $x$ (see e.g. Nagel [14, A-II, Section 2] or Clément, Heijmans et al. [3, Appendix A.1]).

$$
\begin{aligned}
d \Phi(x): & =\left\{x^{*} \in X^{*}:\left\langle y, x^{*}\right\rangle \leq \Phi(y) \text { for all } y \in X, \text { and }\left\langle x, x^{*}\right\rangle=\Phi(x)\right\} \\
& =\left\{x^{*} \in X^{*}:\left\langle y, x^{*}\right\rangle \leq \operatorname{dist}\left(y, X_{+}\right) \text {for all } y \in X, \text { and }\left\langle x, x^{*}\right\rangle=\operatorname{dist}\left(x, X_{+}\right)\right\} \\
& =\left\{x^{*} \in X^{*}:\left\|x^{*}\right\| \leq 1,\left\langle x, x^{*}\right\rangle=0 \text { and }-x^{*} \geq 0\right\} .
\end{aligned}
$$

Since $x \geq 0$ we have

$$
\lim _{h \downarrow 0} \frac{1}{h} \operatorname{dist}\left(x+h F(x), X_{+}\right)=\lim _{h \downarrow 0} \frac{1}{h}(\Phi(x+h F(x))-\Phi(x))=D_{F(x)}^{+}(x)
$$

where $D_{F(x)}^{+}(x)$ denotes the right sided Gateaux-derivative of $\Phi$ at $x$ in the direction of $F(x)$. It is well known (see e.g. Clément, Heijmans et al. [3, Proposition A.1.24]) that

$$
D_{F(x)}^{+} \Phi(x)=\sup \left\{\left\langle F(x), x^{*}\right\rangle: x^{*} \in d \Phi(x)\right\} .
$$

From the explicit form of $d \Phi(x)$ we now conclude that condition (i) is equivalent to $\left\langle F(x), x^{*}\right\rangle \leq 0$ for all $x^{*} \in d \Phi(x)$. By (4.1) this is equivalent to $D_{F(x)}^{+} \Phi(x) \leq 0$, and thus to $\lim _{h \downarrow 0} \frac{1}{h} \operatorname{dist}\left(x+h F(x), X_{+}\right) \leq 0$, hence to condition (ii).

Note that for $x \in \partial X_{+}$the elements $x^{*} \in X^{*}$ with $\left\langle x, x^{*}\right\rangle=\sup _{y \in X_{+}}\left\langle y, x^{*}\right\rangle$ can be interpreted as the normal vectors to $X_{+}$in $x$. Condition (i), or equivalently (ii), is also called the subtangential condition of $F$ in $x \in \partial X_{+}$(compare Deimling [4]). 
The following lemma can be found in Martin [11, Lemma 1.3, p.326].

Lemma 4.5. Let $x \in X_{+}$. Assume that one of the equivalent conditions of Lemma 4.4 holds. Then

$$
\frac{1}{h}\left[T_{0}(h) x+\int_{0}^{h} T_{0}(s) F(x) d s\right]_{-} \rightarrow 0 \text { as } h \downarrow 0 .
$$

We are now ready to prove Theorem 4.2 .

Proof of Theorem 4.2. First we observe that we may, thanks to the timescaling transformation, restrict to the case that $\gamma \equiv 1$ : note that $\gamma$ does not destroy the positive-off-diagonal property (POD). Furthermore we may assume without loss of generality that

$$
F(x)=F\left(x_{+}\right) \text {for all } x \in X .
$$

If this is not satisfied we define $F_{0}: X \rightarrow X$ by $F_{0}(x):=F\left(x_{+}\right)(x \in X)$. Then by construction $F_{0}(x)=F_{0}\left(x_{+}\right)$. Furthermore (POD) remains valid. If solutions of $\left(\mathrm{VOC}_{t}\right)$ with $F$ replaced by $F_{0}$ are positivity preserving, then they coincide with solutions of the original $\left(\mathrm{VOC}_{t}\right)$ for positive initial data $x$.

We first consider the case where $\left\|T_{0}(t)\right\| \leq M e^{\omega t}$ with $M=1$ for all $t \geq 0$ (sometimes called the quasi-contractive case). In a second step we wil reduce the general case (with $M>1$ ) to this situation. Let $x \geq 0$ and let $u(t)=u(t ; x)$ be the continuous solution of $\left(\operatorname{VOC}_{t}\right)$ on $\left[0, t_{\max }(x)\right)$. We show that $u(t) \geq 0$ or equivalently that $u_{-}(t):=[u(t)]_{-}$is zero. For $t<t_{\max }$ we define

$$
\phi(t):=e^{-\omega t}\left\|u_{-}(t)\right\| .
$$

Now

$$
u(t+h)=T_{0}(h) u(t)+\int_{0}^{h} T_{0}(h-s) F(u(t+s)) d s .
$$

Thus by Lemma $4.3(\mathrm{c})$, Lemma 4.5 and (4.3) we have

$$
\begin{aligned}
\left\|u_{-}(t+h)\right\| \leq & \left\|u(t+h)-T_{0}(h) u_{+}(t)-\int_{0}^{h} T_{0}(h-s) F\left(u_{+}(t+s)\right) d s\right\| \\
& +\left\|\left[T_{0}(h) u_{+}(t)+\int_{0}^{h} T_{0}(h-s) F\left(u_{+}(t+s)\right) d s\right]-\right\| \\
\leq & \left\|T_{0}(h) u_{-}(t)\right\|+\left\|u(t+h)-T_{0}(h) u(t)-\int_{0}^{h} T_{0}(h-s) F(u(t+s)) d s\right\| \\
& \quad\left\|\left[T_{0}(h) u_{+}(t)+\int_{0}^{h} T_{0}(h-s) F\left(u_{+}(t)\right) d s\right]_{-}\right\|+o(h) \\
\leq & e^{\omega h}\left\|u_{-}(t)\right\|+o(h) .
\end{aligned}
$$

Hence $\phi(t+h) \leq \phi(t)+o(h)$ for $h \downarrow 0$ and $t<t_{\max }$. In other words

$$
D_{+} \phi(t):=\liminf _{h \downarrow 0} \frac{1}{h}(\phi(t+h)-\phi(t)) \leq 0 .
$$


Since $\phi(0)=\left\|u_{-}(0)\right\|=\left\|x_{-}\right\|=0$, a well known result from the theory of differential inequalities (see e.g. Martin [11, Lemma 7.4, p.260]) implies $\phi=0$.

It remains to consider the case where $\left\|T_{0}(t)\right\| \leq M e^{\omega t}$ with $M>1$. We use a renormalization of $X$ which allows us to reduce this situation to the case $M=1$. For $x \in X$ let $\|x\|^{\prime}:=\sup _{t \geq 0} e^{-\omega t}\left\|T_{0}(t)|x|\right\|$. We have $\|x\| \leq\|x\|^{\prime} \leq M\|x\|$, thus $\|\cdot\|$ and $\|\cdot\|^{\prime}$ are equivalent norms and

$$
\begin{aligned}
\left\|T_{0}(t) x\right\|^{\prime} & =\sup _{s \geq 0} e^{-\omega s}\left\|T_{0}(s)\left|T_{0}(t) x\right|\right\| \leq \sup _{s \geq 0} e^{-\omega s}\left\|T_{0}(s+t)|x|\right\| \\
& \leq e^{\omega t} \sup _{s \geq 0} e^{-\omega(s+t)}\left\|T_{0}(t+s)|x|\right\| \leq e^{\omega t} \sup _{s \geq 0} e^{-\omega s}\left\|T_{0}(s)|x|\right\|=e^{\omega t}\|x\|^{\prime} .
\end{aligned}
$$

Let $x, y \in X$ with $|x| \leq|y|$, then $\||x|\|^{\prime}:=\sup _{t \geq 0} e^{-\omega t}\left\|T_{0}(t)|x|\right\| \leq$ $\sup _{t \geq 0} e^{-\omega t}\left\|T_{0}(t)|y|\right\|=\||y|\|^{\prime}$. This shows that $\|\cdot\|^{\prime}$ defines a lattice norm on $X$. Hence the properties listed in Lemma 4.3 hold for $\|\cdot\|^{\prime}$ and dist' (dist' defined in the obvious way) as well. Thus $\left\{T_{0}(t), t \geq 0\right\}$ is a positive quasicontraction semigroup on $\left(X,\|\cdot\|^{\prime}\right)$ and we are in the before discussed situation and thus the assertion follows.

Note that our perturbation $F$ needs not to be defined on the whole space $X$ but only on $X_{+}$, which is of course the situation we usually meet in biological examples.

Another important property of solutions of $\left(\mathrm{P}_{t}\right)$ or $\left(\mathrm{VOC}_{t}\right)$ which has to be investigated is the boundedness. Recall from Theorem 3.2 that solutions of $\left(\mathrm{VOC}_{t}\right)$ which are bounded on a finite time interval can be extended. Thus in order to obtain global existence of a solution of $\left(\mathrm{VOC}_{t}\right)$, we have to show that it stays bounded on any finite time interval.

Theorem 4.6. Let $A_{0}$ be generator of a linear bounded positive $C_{0}$-semigroup $\left\{T_{0}(t), t \geq 0\right\}$. Assume that $u(t ; x) \geq 0$ if $x \geq 0$ and $t<t_{\max }(x)$. Furthermore let $F$ satisfy the following "off-diagonal-boundedness" property: there exists an operator $F_{0}$ on $X$ such that

$$
\begin{array}{ll}
\text { (i) } & F(x) \leq F_{0}(x) \text { for all } x \geq 0 \\
\text { (ii) } & \left\|F_{0}(x)\right\| \leq C\|x\| \text { for all } x \geq 0 .
\end{array}
$$

Then $t_{\max }(x)=\infty$ for all $x \geq 0$.

Proof. In Section 3 we have seen that a mild solution $u(t)=u(t ; x)$ of $\left(\mathrm{P}_{t}\right)$ is by definition a continuous solution of the variation-of-constants formula

$$
u(t)=T_{0}\left(\tau_{u}(t)\right) x+\int_{0}^{t} T_{0}\left(\tau_{u}(t)-\tau_{u}(s)\right) F(u(s)) d s, \quad t \in\left[0, t_{0}\right),
$$

where $\tau_{u}$ is given by formula (2.1). Let $x \in X, x \geq 0$ and $t<t_{0}$. Using assumption (i) for $F$ and the positivity of $u(s)$ we have

$$
u(t) \leq T_{0}\left(\tau_{u}(t)\right) x+\int_{0}^{t} T_{0}\left(\tau_{u}(t)-\tau_{u}(s)\right) F_{0}(u(s)) d s
$$


since every $T_{0}(t)$ is a positive operator. Thus by assumption (ii) and the boundedness of the semigroup $\left\{T_{0}(t), t \geq 0\right\}$ there exists a constant $M>0$ such that

$$
\|u(t)\| \leq M\|x\|+\int_{0}^{t} M C\|u(s)\| d s .
$$

By the lemma of Gronwall we have $\|u(t)\| \leq M \cdot e^{M C t_{0}}\|x\|$ for all $t<t_{0}$. We may therefore conclude that there is a globally defined continuous solution of $\left(\mathrm{VOC}_{t}\right)$ or, equivalently, a global mild solution of $\left(\mathrm{P}_{t}\right)$.

REMARK 4.7. (a) To assume that $\|F(x)\| \leq M\|x\|$ for all $x \geq 0$ is much stronger than the off-diagonal-boundedness property (4.4). Indeed in many examples from structured population dynamics such an assumption is not very realistic due to the presence of a death term, whereas the existence of an operator $F_{0}$ as in (4.4) can usually be established.

(b) One can easily construct examples which show that the assumption " $\left\{T_{0}(t), t \geq 0\right\}$ bounded" is essential in order to have global solutions of $\left(\mathrm{P}_{t}\right)$.

Corollary 4.8. Let $A_{0}$ be the infinitesimal generator of a linear, positive, bounded $C_{0}$-semigroup $\left\{T_{0}(t), t \geq 0\right\}$. Furthermore, assume that $F$ satisfies the positive-off-diagonal property (POD) and the off-diagonal-boundedness property (4.4). Then $t_{\max }(x)=\infty$ for all $x \geq 0$.

\section{Principle of Linearized (In) stability}

In this section we prove that the principle of linearized (in)stability holds for quasilinear equations of the form $\left(\mathrm{P}_{t}\right)$. Obviously an equilibrium $\bar{u}$ of $\left(\mathrm{P}_{t}\right)$ is an equilibrium of $\left(\mathrm{P}_{\tau}\right)$, and vice versa. The main result of this section is the following.

TheOrem 5.1. Let $\bar{u}$ be an equilibrium of $\left(\mathrm{P}_{t}\right)$. Then it is stable for $\left(P_{t}\right)$ if and only if it is stable for $\left(\mathrm{P}_{\boldsymbol{\tau}}\right)$.

Proof. Without loss of generality we may assume that $\bar{u}=0$ which means that $F(0)=0$. We only prove the if part. The only if part is proved in the same way.

Assume that 0 is a stable equilibrium of $\left(\mathrm{P}_{\tau}\right)$. Let $\epsilon>0$. We must show that there is a $\delta>0$ such that for $\|x\| \leq \delta$ :

(i) $t_{\max }(x)=\infty$, and

(ii) $\|u(t ; x)\| \leq \epsilon, \quad t \geq 0$.

By hypothesis there exists $\delta>0$ such that for $\|x\| \leq \delta$ :

(i') $\tau_{\max }(x)=\infty$, and

(ii') $\|v(\tau ; x)\| \leq \epsilon, \quad \tau \geq 0$.

Recall that

$$
t_{\max }(x)=\int_{0}^{\infty} \frac{d \sigma}{\gamma(v(\sigma ; x))} .
$$


Since $\gamma$ is locally bounded there exists $\eta>0$ such that for all $v$ with $\|v\| \leq \epsilon$ we have $\gamma(v) \leq \eta$. Hence, if $\|x\| \leq \delta$ we have

$$
t_{\max }(x) \geq \int_{0}^{\infty} \frac{d \sigma}{\eta}=\infty .
$$

Furthermore,

$$
\|u(t ; x)\|=\|v(\tau(t ; x) ; x)\| \leq \epsilon, \quad t \geq 0 .
$$

Thus we have proved (i) and (ii).

Motivated by this result we first collect some results concerning linearized stability for the semilinear case. So we assume for the moment that $\gamma \equiv 1$ (and hence $F=B$ ), and consider the Cauchy problem

$$
\begin{aligned}
& \frac{d}{d t} u(t)=A_{0} u(t)+B(u(t)), \\
& u(0)=x
\end{aligned}
$$

We restrict our attention to the case where 0 is an equilibrium solution of (5.1), thus $B(0)=0$. Furthermore we assume without loss of generality that $\left\|T_{0}(t)\right\| \leq 1$ for all $t \geq 0$.

For $x \in X$ let $u(t):=u(t ; x)$ be the local solution of $(5.1)$ defined for $t<$ $t_{\max }(x)$. First we show, that for any given time $t_{0}$ the solution $u(t ; x)$ exists up to time $t_{0}$ if $x$ is close enough to the equilibrium 0 . Recall that $\mathcal{C}(r)$ is the Lipschitz constant of $B$ : see Section 3 .

Proposition 5.2. Assume that $B(0)=0$. Let $x \in X$ and let $u(t):=u(t ; x)$ be a mild solution of (5.1), hence a continuous solution to the corresponding variationof-constants equation.

(i) If $r \in \mathbb{R}_{+}$and $\|x\| \leq r$, then $\|u(t)\| \leq e^{t \mathcal{C}(r)}\|x\|$, whenever $t \leq(\mathcal{C}(r))^{-1} \log (r$. $\left.\|x\|^{-1}\right)$.

(ii) For all $t_{0} \geq 0$ there exists $r>0$ such that $t_{\max }(x) \geq t_{0}$ for all $x$ with $\|x\| \leq r$. Then

Proof. (i): Let $\|x\| \leq r$ and let $t \geq 0$ be such that $\|u(s)\| \leq r$ for all $0 \leq s \leq t$.

$$
\|u(t)\| \leq\|x\|+\int_{0}^{t}\|B(u(s))\| d s \leq\|x\|+\int_{0}^{t} \mathcal{C}(r)\|u(s)\| d s .
$$

Using Gronwall's lemma we obtain $\|u(t)\| \leq e^{t \mathcal{C}(r)}\|x\|$. Let $t=\inf \{s>0 \mid\|u(s)\|=$ $r\}$. Note that $t$ may equal $\infty$. Then, if $t<\infty, r \leq e^{t \mathcal{C}(r)}\|x\|$, hence $t \geq(\mathcal{C}(r))^{-1} \times$ $\log \left(r\|x\|^{-1}\right)$. From this the result follows immediately.

(ii): Let $t_{0} \geq 0$ and define $r$ by $r:=e^{-t_{0} \mathcal{C}(1)}$. For $\|x\| \leq r \leq 1$ we obtain by part (i) that $\|u(s)\| \leq e^{s \mathcal{C}(1)}\|x\|$ whenever $s \leq(\mathcal{C}(1))^{-1} \log \left(\|x\|^{-1}\right)$. Hence $\|u(s)\| \leq$ $e^{s \mathcal{C}(1)}\|x\|$ for $s \leq t_{0}=(\mathcal{C}(1))^{-1} \log \left(r^{-1}\right)$. This implies $t_{\max }(x)>t_{0}$. 
Now assume that $B$ is Fréchet differentiable and denote its Fréchet derivative at 0 by $L:=(D B)(0)$, which is a bounded linear operator mapping $X$ into $X$. We define

$$
H(u):=B(u)-L u \text {. }
$$

Let $\left\{T_{L}(t), t \geq 0\right\}$ be the perturbed linear $C_{0}$-semigroup generated by $A_{0}+L$. Since $\left\{T_{0}(t), t \geq 0\right\}$ is a contraction semigroup there exists $\omega>0$, such that $\left\|T_{L}(t)\right\| \leq e^{\omega t}$ for all $t \geq 0$. We shall prove that for every $t \geq 0, x \mapsto u(t ; x)$ is Fréchet differentiable at $x=0$ and that

$$
\left(D_{x} u(t ; x)\right)(0)=T_{L}(t)
$$

We use the following identity which is a consequence of Clément et al. [2, Part III, Proposition 2.5]:

$$
u(t ; x)-T_{L}(t) x=\int_{0}^{t} T_{L}(t-s) H(u(s ; x)) d s \text { for } t<t_{\max }(x) .
$$

Theorem 5.3. For every $t \geq 0$ the mapping $x \mapsto u(t ; x)$ is Fréchet differentiable at 0 and its derivative equals $T_{L}(t)$.

Proof. Fix $t \geq 0$. For all $\varepsilon>0$ we have to find $\delta>0$ such that $\| u(t ; x)-$ $T_{L}(t) x\|\leq \varepsilon\| x \|$ whenever $\|x\| \leq \delta$. Choose $0<\delta<1$ such that

$$
\begin{aligned}
& \delta \leq e^{-\mathcal{C}(1) t}, \quad \text { and } \\
& \|H(u)\| \leq \varepsilon \mathcal{C}(1) e^{-(\omega+\mathcal{C}(1)) t}\|u\| \text { whenever }\|u\| \leq e^{t \mathcal{C}(1)} \delta .
\end{aligned}
$$

Now fix $x \in X$ with $\|x\|<\delta$. For short we write $u(t):=u(t ; x)$. By Proposition $5.2(\mathrm{i})$ we have

$$
\|u(s)\| \leq e^{s \mathcal{C}(1)}\|x\| \quad \text { if } s \leq \mathcal{C}(1)^{-1} \log \left(\|x\|^{-1}\right)
$$

hence by $(*)$

$$
\|u(s)\| \leq e^{s \mathcal{C}(1)}\|x\| \quad \text { if } s \leq t
$$

Using $(* *)$ we obtain

$$
\|H(u(s))\| \leq \varepsilon \mathcal{C}(1) e^{-(\omega+\mathcal{C}(1)) t} e^{s C(1)}\|x\| \quad \text { if } s \leq t,
$$

and thus by (5.4)

$$
\begin{aligned}
\left\|u(t)-T_{L}(t) x\right\| & \leq \int_{0}^{t} e^{\omega(t-s)} \varepsilon \mathcal{C}(1) e^{-(\omega+\mathcal{C}(1)) t} e^{s \mathcal{C}(1)}\|x\| d s \\
& \leq \varepsilon \mathcal{C}(1) e^{-\mathcal{C}(1) t}\|x\| \int_{0}^{t} e^{s \mathcal{C}(1)} d s \leq \varepsilon\|x\| .
\end{aligned}
$$

As usual we denote by $\omega\left(A_{0}+L\right)$ the growth rate (type) of the semigroup $\left\{T_{L}(t), t \geq 0\right\}$. 
Theorem 5.4. Let $\omega\left(A_{0}+L\right)<0$ and $0 \leq \nu<-\omega\left(A_{0}+L\right)$. Then there exists $\delta>0$ such that for $\|x\| \leq \delta$ we have $t_{\max }(x)=\infty$ and $\lim _{t \rightarrow \infty} e^{\nu t}\|u(t ; x)\|=0$.

Proof. We take $\nu=0$. (If $\nu \neq 0$ consider $e^{\nu t} T_{L}(t)$ instead of $T_{L}(t)$.) Let $t_{0}>0$ such that $\left\|T_{L}\left(t_{0}\right)\right\| \leq \frac{1}{4}$. Furthermore choose $\delta_{1} \in(0,1)$ such that for $\|x\| \leq \delta_{1}$ we have

$$
\begin{aligned}
& t_{\max }(x) \geq t_{0} \\
& \left\|u\left(t_{0} ; x\right)-T_{L}\left(t_{0}\right) x\right\| \leq \frac{1}{4}\|x\| .
\end{aligned}
$$

Define $\delta:=e^{-t_{0} \mathcal{C}(1)} \delta_{1}$. If $\|x\| \leq \delta_{1}$, then $t_{\max }(x) \geq t_{0}$ and

$$
\left\|u\left(t_{0} ; x\right)\right\| \leq\left\|u\left(t_{0} ; x\right)-T_{L}\left(t_{0}\right) x\right\|+\left\|T_{L}\left(t_{0}\right) x\right\| \leq \frac{1}{2}\|x\| \leq \frac{1}{2} \delta_{1} .
$$

Hence $t_{\max }\left(u\left(t_{0} ; x\right)\right) \geq t_{0}$ and thus $t_{\max }(x) \geq 2 t_{0}$ and

$$
\left\|u\left(2 t_{0} ; x\right)\right\| \leq\left(\frac{1}{2}\right)^{2}\|x\| .
$$

By iteration we find that for every $n \geq 0$ we have

$$
t_{\max }(x) \geq n t_{0} \quad \text { and } \quad\left\|u\left(n t_{0} ; x\right)\right\| \leq 2^{-n}\|x\| .
$$

In particular this implies that $t_{\max }(x)=\infty$.

Now let $\|x\| \leq \delta \leq \delta_{1}$. Then, as before, $t_{\max }(x)=\infty$. For every $t \geq 0$ we have $t=n t_{0}+t_{1}$, where $t_{1} \in\left[0, t_{0}\right)$. Thus

$$
\|u(t ; x)\|=\left\|u\left(n t_{0} ; u\left(t_{1} ; x\right)\right)\right\| \leq 2^{-n}\left\|u\left(t_{1} ; x\right)\right\|,
$$

since $\left\|u\left(t_{1} ; x\right)\right\| \leq e^{t_{1} \mathcal{C}(1)}\|x\|<e^{t_{0} \mathcal{C}(1)} \delta=\delta_{1}$ by Proposition 5.2(i), the choice of $t_{1}$ and the definition of $\delta$. Consequently

$$
\|u(t ; x)\| \leq 2^{-n} e^{t_{1} \mathcal{C}(1)}\|x\| \quad \text { for all } t \geq 0 \text { and all } n \in N
$$

hence $\lim _{t \rightarrow \infty}\|u(t ; x)\|=0$.

The corresponding instability result is the following.

Theorem 5.5. Let $X=X_{1} \oplus X_{2}$ where $X_{1}$ and $X_{2}$ are invariant under $T_{L}(t)$ and $\operatorname{dim} X_{1}<\infty$. Let $T_{i}(t)$ denote the restriction of $T_{L}(t)$ to $X_{i}(i=1,2)$ and $A_{i}$ the corresponding generator. Finally assume that

$$
\omega\left(A_{2}\right)<\min \left\{\operatorname{Re} \lambda \mid \lambda \in \sigma\left(A_{1}\right)\right\}
$$

and that

$$
0<s\left(A_{1}\right):=\max \left\{\operatorname{Re} \lambda \mid \lambda \in \sigma\left(A_{1}\right)\right\}
$$


Then there exists an $\varepsilon>0$, a sequence $t_{n}$ in $\boldsymbol{R}_{+}, t_{n} \rightarrow \infty$, and a sequence $x_{n}$ in $X, x_{n} \rightarrow 0$, such that $t_{\max }\left(x_{n}\right)>t_{n}$ and

$$
\left\|u\left(t_{n} ; x_{n}\right)\right\| \geq \varepsilon
$$

for $n$ large enough.

Proof. Let $P$ denote the projection on $X_{1}$ and let $Q=I-P$ be the projection on $X_{2}$. Without loss of generality we may assume that

$$
\|x\|=\|P x\|+\|Q x\|, \quad \text { for } x \in X .
$$

(Otherwise one can consider the equivalent norm $\|x\|_{e}:=\|P x\|+\|Q x\|$.)

Furthermore we suppose that

$$
\min \left\{\operatorname{Re} \lambda: \lambda \in \sigma\left(A_{1}\right)\right\}>0 .
$$

This can always be achieved by taking $X_{1}$ small enough. Hence there exists $\mu>0$ such that

$$
\left\|T_{L}(t) x_{1}\right\| \geq e^{\mu t}\left\|x_{1}\right\| \quad \text { for } t \geq 0 \text { and } x_{1} \in X_{1} .
$$

Let $0<\eta<\mu$ be such that

$$
\left\|T_{L}(t) x_{2}\right\| \leq e^{\eta t}\left\|x_{2}\right\| \quad \text { for } t \geq 0 \text { and } x_{2} \in X_{2} .
$$

Let $t_{0}>0$ be fixed and define

$$
\sigma:=-\frac{1}{t_{0}} \log \left(\frac{e^{\mu t_{0}}-e^{\eta t_{0}}}{2}\right)
$$

By Theorem 5.3 there exists an $\varepsilon>0$ such that

$$
\left\|T_{L}\left(t_{0}\right) x-u\left(t_{0} ; x\right)\right\| \leq \frac{1}{2} e^{-\sigma t_{0}}\|x\| \quad \text { if }\|x\| \leq \varepsilon .
$$

Let $x$ be such that $\|x\| \leq \varepsilon$ and $\|Q x\| \leq\|P x\|$. Assume that

$$
\left\|u\left(m t_{0} ; x\right)\right\|<\varepsilon \text { for all } m \in \mathbf{N} .
$$

(In particular $t_{\max }(x)=\infty$.)

Then we obtain

$$
\begin{aligned}
\left\|P u\left(t_{0} ; x\right)\right\| & \geq\left\|P T_{L}\left(t_{0}\right) x\right\|-\left\|P\left(u\left(t_{0} ; x\right)-T_{L}\left(t_{0}\right) x\right)\right\| \\
& \geq e^{\mu t_{0}}\|P x\|-\left\|u\left(t_{0} ; x\right)-T_{L}\left(t_{0}\right) x\right\| \\
& \geq e^{\mu t_{0}}\|P x\|-\frac{1}{2} e^{-\sigma t_{0}}\|x\| \\
& \geq e^{\mu t_{0}}\|P x\|-\frac{1}{2} e^{-\sigma t_{0}}(\|P x\|+\|Q x\|) \\
& \geq e^{\mu t_{0}}\|P x\|-e^{-\sigma t_{0}}\|P x\|
\end{aligned}
$$


and also

$$
\begin{aligned}
\left\|Q u\left(t_{0} ; x\right)\right\| & \leq\left\|Q T_{L}\left(t_{0}\right) x\right\|+\left\|Q\left(u\left(t_{0} ; x\right)-T_{L}\left(t_{0}\right) x\right)\right\| \\
& \leq\left\|Q T_{L}\left(t_{0}\right) x\right\|+\left\|u\left(t_{0} ; x\right)-T_{L}\left(t_{0}\right) x\right\| \\
& \leq e^{\eta t_{0}}\|Q x\|+\frac{1}{2} e^{-\sigma t_{0}}\|x\| \\
& \leq e^{\eta t_{0}}\|Q x\|+e^{-\sigma t_{0}}\|P x\| \\
& \leq\left(e^{\eta t_{0}}+e^{-\sigma t_{0}}\right)\|P x\| \\
& =\left(e^{\mu t_{0}}-e^{-\sigma t_{0}}\right)\|P x\| \text { by }(*) .
\end{aligned}
$$

Consequently $\left\|P u\left(t_{0} ; x\right)\right\| \geq\left\|Q u\left(t_{0} ; x\right)\right\|$ and by iteration we now find that

$$
\left\|P u\left(n t_{0} ; x\right)\right\| \geq\left(e^{\eta t_{0}}+e^{-\sigma t_{0}}\right)^{n}\|P x\|,
$$

hence $\left\|P u\left(n t_{0} ; x\right)\right\| \rightarrow \infty$ since $\eta>0$. So

$$
\left\|u\left(n t_{0} ; x\right)\right\|=\left\|P u\left(n t_{0} ; x\right)\right\|+\left\|Q u\left(n t_{0} ; x\right)\right\| \rightarrow \infty \text { for } n \rightarrow \infty .
$$

This yields a contradiction to $(* *)$ and the result follows.

We return to our original quasilinear problem $\left(\mathrm{P}_{t}\right)$. By $u(t ; x)$ we again denote solutions of $\left(\mathrm{P}_{t}\right)$. From Theorems 5.4 and 5.5 we know that (in)stability of the equilibrium 0 of the semilinear problem $\left(\mathrm{P}_{\tau}\right)$ and therefore of the quasilinear problem $\left(\mathrm{P}_{t}\right)$, hinges upon the spectral properties of the generator $A_{0}+B^{\prime}(0)$. Here we have assumed that $B=F / \gamma$ is Fréchet differentiable at 0 . If both $F$ and $\gamma$ are differentiable at 0 , then the linearization of $\left(\mathrm{P}_{t}\right)$ at $u=0$ is given by

$$
\frac{d w}{d t}=\gamma(0) A_{0} w+F^{\prime}(0) w .
$$

Since

$$
\gamma(0) A_{0}+F^{\prime}(0)=\gamma(0)\left(A_{0}+B^{\prime}(0)\right)
$$

and $\gamma(0)>0$ we may equivalently state that the stability of the equilibrium is determined by (5.5), or more precisely, by the spectral properties of the linearized operator $\gamma(0) A_{0}+F^{\prime}(0)$.

Let us denote by $\{S(t), t \geq 0\}$ the semigroup corresponding to the linear Cauchy problem (5.5). One might wonder if the extension of Theorem 5.3 for $\gamma \neq \equiv 1$ holds: is $x \mapsto u(t ; x)$ Fréchet differentiable at $x=0$ with derivative $S(t)$ ? We now present an example which shows that this is not true in general.

EXAMPLE 5.6. Let $S^{1}$ be the unit circle in $\boldsymbol{R}^{2}$ and $X=C\left(S^{1}\right)$. We will identify $S^{1}$ with $\boldsymbol{R} /[0,2 \pi)$. Define the $C_{0}$-group $\left\{T_{0}(t), t \geq 0\right\}$ on $X$ by:

$$
\left(T_{0}(t) x\right)(\theta):=x(\theta-t), \quad \theta \in S^{1}, t \in \boldsymbol{R} .
$$

Let $\gamma(x):=1 / 2+|1 / 2+x(0)|$ and $F \equiv 0$. Obviously, $x=0$ is a stable equilibrium of

$$
\frac{d u}{d t}=\gamma(u) A_{0} u, \quad u(0)=x
$$


Note that $\gamma(u)$ represents the speed of rotation along the circle. Obviously, $\gamma$ is differentiable at $u=0$ and the linearization around 0 is given by

$$
\frac{d w}{d t}=A_{0} w
$$

Let $\{T(t), t \geq 0\}$ be the nonlinear semigroup generated by (5.6) (note that this problem has a global solution for every $x \in X$ ).

(a)

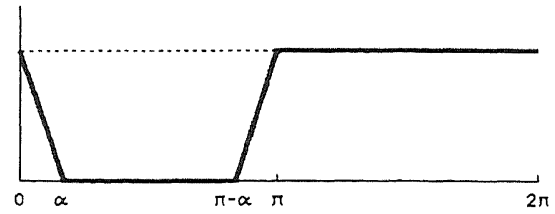

(b)

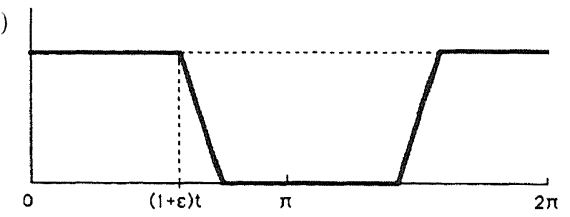

(c)

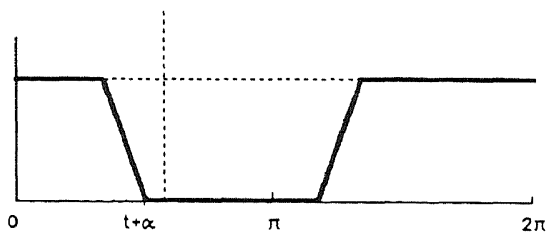

We show that $T_{0}(t)$ is not the Fréchet derivative of $x \mapsto T(t) x$ at $x=0$. Actually we prove more, namely that for some $t>0$ (but one can extend this to all $t>0$ ) the following holds: for all $\epsilon$ with $0<\epsilon<1$ there exists $x \in X$ with $\|x\|=\epsilon$, such that $\left\|T(t) x-T_{0}(t) x\right\|=\|x\|$. Namely, fix $t \in(0, \pi / 2]$ and $0<\epsilon<1$. Choose $\alpha>0$ such that $\alpha \leq \epsilon t$.

Let $x$ be as depicted in Figure (a). During the time interval $[0, t]$ the rotation velocity in (5.6) equals

$$
\gamma(u(t ; x))=1 / 2+|1 / 2+u(t ; x)(0)|=1+\epsilon
$$

since $u(t ; x)(0)=(T(t) x)(0)=\epsilon$ for all $t \in(0, \pi / 2]$, whereas this speed is constantly 1 in (5.7). Therefore $T(t) x$ and $T_{0}(t) x$ are as depicted in Figure (b) and (c) respectively. Since $\alpha \leq \epsilon t$ we have $(1+\epsilon) t \geq t+\alpha$ and we find that $\left\|T(t) x-T_{0}(t) x\right\|=$ $\epsilon=\|x\|$.

For the sake of completeness we want to conclude this section by mentioning the explicit formula for the linearization of $\left(\mathrm{P}_{t}\right)$ in a nontrivial equilibrium point $\bar{u}$. Again we first consider the linearized equation corresponding to $\left(\mathrm{P}_{\tau}\right)$. We obtain:

$$
\begin{aligned}
\frac{d w}{d t} & =A_{0} w+\frac{1}{\gamma(\bar{u})} F^{\prime}(\bar{u}) w-\frac{\left\langle\gamma^{\prime}(\bar{u}), w\right\rangle}{\gamma(\bar{u})^{2}} F(\bar{u}) \\
& =A_{0} w+\left\langle\gamma^{\prime}(\bar{u}), w\right\rangle \cdot \frac{1}{\gamma(\bar{u})} A_{0} \bar{u}+\frac{1}{\gamma(\bar{u})} F^{\prime}(\bar{u}) w .
\end{aligned}
$$


This system corresponds to the linearization of $\left(\mathrm{P}_{t}\right)$ in $\bar{u}$ which is given by:

$$
\frac{d w}{d t}=\gamma(\bar{u}) A_{0} w+\left\langle\gamma^{\prime}(\bar{u}), w\right\rangle A_{0} \bar{u}+F^{\prime}(\bar{u}) w
$$

\section{Application to the Cell Division Model}

After having studied the general quasilinear equation in some detail we now return to our starting point: the model describing size-dependent cell growth and division described by equation (1.2). Before starting the analysis we have to specify the setting. Let us recall from Section 1 the initial value problem which we want to investigate:

$$
\begin{aligned}
& \begin{aligned}
\frac{\partial}{\partial t} p(t, s)+\widehat{\gamma}(E(t)) \frac{\partial}{\partial s}(g(s) p(t, s))= & -\mu(s) p(t, s)-b(s) p(t, s) \\
& +4 b(2 s) p(t, 2 s)
\end{aligned} \\
& g(\alpha / 2) p(t, \alpha / 2)=0 \\
& \frac{d}{d t} E(t)=-\sigma E(t)+\int_{\alpha / 2}^{1} h(s) p(t, s) d s \\
& p(0, s)=p_{0}(s) \geq 0 \\
& E(0)=E_{0} \geq 0 .
\end{aligned}
$$

The biological interpretation of this equation, outlined in Section 1, suggests to look for densities $p$ satisfying $(6.1)$ and hence for solutions $p(t, \cdot) \in L^{1}([\alpha / 2,1])$ which are positive.

We assume that the minimal possible cell division size of a cell is $\alpha$. Thus the possible cell size $s$ of an individual cell is restricted to values between a minimal size $\alpha / 2$ and a maximal size 1 . Furthermore we make the following assumptions on $\widehat{\gamma}, g, \mu, b$ and $h$ :

Assumption 6.1.

$\left(\mathrm{A}_{\widehat{\gamma}}\right) \widehat{\gamma}: X \rightarrow \boldsymbol{R}_{+} \backslash\{0\}$ is locally bounded and Fréchet differentiable.

$\left(\mathrm{A}_{g}\right) g:[\alpha / 2,1] \rightarrow \boldsymbol{R}_{+} \backslash\{0\}$ is continuous.

$\left(\mathrm{A}_{\mu}\right) \mu:[\alpha / 2,1] \rightarrow \boldsymbol{R}_{+}$is measurable and bounded.

$\left(\mathrm{A}_{b}\right) b:[\alpha / 2,1] \rightarrow \mathbb{R}_{+}$is measurable and bounded, $b(x)=0$ for a.e. $x \in$ $[\alpha / 2, \alpha]$ and $b(x)>0$ for a.e. $x \in(\alpha, 1]$.

$\left(\mathrm{A}_{h}\right) h:[\alpha / 2,1] \rightarrow \boldsymbol{R}_{+}$is measurable and bounded.

REMARK. In $\left(A_{b}\right)$ we assume that the division rate $b$ is bounded on $[\alpha / 2,1]$. The biological interpretation of this mathematical assumption has quite drastic consequences for our model. The boundedness of $b$ implies that a cell which does not divide before reaching size 1 will never divide. It will just exist and grow until it eventually dies. Nevertheless these large, quiescent cells may have an effect on 
our system (6.1), since we assumed that the total number of cells (weighted by $h$ ) will contribute to the production of the enzyme $E$. Although cells of size $s>1$ may be relevant for the system, they are not included in our model formulation. There are at least two ways out of the dilemma. We could make the assumption that cells with size $s>1$ will automatically belong to another type of cells (for example by differentiation), which is out of our consideration. Thus they have no (direct) influence on the amount of the enzyme $E$ present. Alternatively we could assume that cells which reach the threshold $s=1$ die instantaneously. This may seem rather drastic but one should realize that the fraction of cells which indeed reaches this size can be negligible small depending on $b$ and $\mu$.

The system (6.1) is of the form $\left(\mathrm{P}_{t}\right)$. To show this we have to define $A_{0}, F$ and $\gamma$.

Let $X:=L^{1}[\alpha / 2,1] \times \boldsymbol{R}$. We define $\gamma, A_{0}$ and $F$ by

$$
\begin{aligned}
& \gamma((p, E)):=\widehat{\gamma}(E), \\
& A_{0}((p, E)):=\left(-(g p)^{\prime}, 0\right),
\end{aligned}
$$

with domain

$$
D\left(A_{0}\right):=\left\{(p, E) \in L^{1}[\alpha / 2,1] \times \mathbb{R} \mid p \in A C[\alpha / 2,1] \text { and } p(\alpha / 2)=0\right\},
$$

and

$$
F((p, E)):=\left(s \mapsto[-\mu(s) p(s)-b(s) p(s)+4 b(2 s) p(2 s)],-\sigma E+\int_{\alpha / 2}^{1} h(s) p(s) d s\right)
$$

where we set $b(2 s) p(2 s)=0$ if $s>1 / 2$.

If we define

$$
u(t):=(p(t, \cdot), E(t)) \quad \text { and } \quad x:=\left(p_{0}(\cdot), E_{0}\right)
$$

we see that (6.1) is of the form $\left(\mathrm{P}_{t}\right)$.

It is obvious that $A_{0}$ generates a bounded, positive $C_{0}$-semigroup $\left\{T_{0}(t), t \geq 0\right\}$ and that Assumptions 2.1 and 3.1 are satisfied. Furthermore one easily verifies that $F / \gamma$ satisfies the positive-off-diagonal property (POD) introduced in Section 4. Since $F$ is linear it automatically satisfies the off-diagonal-boundedness property (4.4). Summarizing we have:

Theorem 6.2. Let Assumption (6.1) hold and suppose that $E_{0} \geq 0$ and $p_{0}$ is a positive, absolutely continuous function satisfying $p_{0}(\alpha / 2)=0$. Then there exists a unique positive, continuously differentiable solution $p(t, \cdot), E(t)$ of $(6.1)$ defined for all $t \geq 0$.

The abstract theory gives us solutions of (6.1) for all initial data $p_{0}$ in $L^{1}[\alpha / 2$, 1]. In general these solutions are not continuously differentiable but satisfy a related integral equation which can be obtained by integration of (6.1) along characteristics. 
Next we will investigate the existence and stability properties of positive equilibrium solutions of the nonlinear system (6.1). Of course $(0,0)$ is an equilibrium point, but in general there may be others. Such nonzero equilibria $(\bar{p}, \bar{E})$ can be obtained from the functional differential equation

$$
\begin{aligned}
& \widehat{\gamma}(\bar{E}) \frac{d}{d s}(g \bar{p})=-\mu(s) \bar{p}(s)-b(s) \bar{p}(s)+4 b(2 s) \bar{p}(2 s), \\
& g(\alpha / 2) \bar{p}(\alpha / 2)=0 \\
& \sigma \bar{E}=\int_{\alpha / 2}^{1} h(s) \bar{p}(s) d s .
\end{aligned}
$$

To make life easy we assume that $\alpha>1 / 2$. Then we can solve (6.3a), (6.3b) successively for $s \geq 1 / 2$ and $s<1 / 2$ and obtain

$$
\bar{p}(s)=c q(s) \text { for all } s \in[\alpha / 2,1],
$$

for a constant $c$ (which must still be determined) and

$$
q(s):= \begin{cases}\frac{H_{\theta}(s)}{g(s)} & \text { if } s \geq 1 / 2, \\ \frac{4}{\theta} \frac{H_{\theta}(s)}{g(s)} \int_{\alpha / 2}^{s} \frac{b(2 \xi)}{g(2 \xi)} \frac{H_{\theta}(2 \xi)}{H_{\theta}(\xi)} & \text { if } s<1 / 2 .\end{cases}
$$

Here $\theta:=\widehat{\gamma}(\bar{E})$ and

$$
H_{\theta}(s):=\exp \left(-\frac{1}{\theta} \int_{\alpha / 2}^{s} \frac{b(\xi)+\mu(\xi)}{g(\xi)} d \xi\right) \text { for all } s \in[\alpha / 2,1] .
$$

Since $\bar{p}$ is an element of $D\left(A_{0}\right)$ it has to be a continuous function, in particular it must be continuous at $s=1 / 2$. From this we obtain a scalar equation which determines $\bar{E}$, or more precisely $\theta=\widehat{\gamma}(\bar{E})$, namely by the fixed point equation:

$$
\theta=Q(\theta)
$$

where

$$
Q(\theta):=4 \int_{\alpha / 2}^{1 / 2} \frac{b(2 \xi)}{g(2 \xi)} \exp \left(-\frac{1}{\theta} \int_{\xi}^{2 \xi} \frac{\mu(\eta)+b(\eta)}{g(\eta)} d \eta\right) d \xi \quad \text { for all } \theta>0 .
$$

We have proved the following result:

THEOREM 6.3. The pair $(\bar{p}, \bar{E}) \neq(0,0)$ is an equilibrium of $(6.1)$ if and only if $\widehat{\gamma}(\bar{E})$ is a solution of $(6.5)$ and $\bar{p}=c q$, with $c:=\sigma \bar{E}\left(\int_{\alpha / 2}^{1} h(s) q(s) d s\right)^{-1}$.

Note that $Q$ is a continuous increasing function and that $Q(0)=0$. The quantity $Q(\theta)$ can be interpreted as the average number of offspring: see Metz and Diekmann [2, Interlude 4.3.2, p.36]. 
From our previous results we know that the local stability properties of an equilibrium are determined by the linearized system:

$$
\begin{aligned}
& \frac{\partial}{\partial t} w(t, s)+\widehat{\gamma}(\bar{E}) \frac{\partial}{\partial s}(g(s) w(t, s))=-\widehat{\gamma}^{\prime}(\bar{E}) \frac{\partial}{\partial s}(g(s) \bar{p}(s)) \cdot \mathcal{E}(t)-\mu(s) w(t, s) \\
&-b(s) w(t, s)+4 b(2 s) w(t, 2 s) \\
& g(\alpha / 2) w(t, \alpha / 2)=0 \\
& \frac{d}{d t} \mathcal{E}(t)=-\sigma \mathcal{E}(t)+\int_{\alpha / 2}^{1} h(s) w(t, s) d s
\end{aligned}
$$

Acknowledgment. We wish to thank Horst Thieme for useful discussions concerning the positivity of solutions of semilinear equations.

\section{References}

[1] J.M. Ball, Strongly continuous semigroups, weak solutions and the variation of constants formula. Proc. Amer. Math. Soc., 63 (1977), 370-373.

[2] Ph. Clément, O. Diekmann, M. Gyllenberg, H.J.A.M. Heijmans and H.R. Thieme, Perturbation theory for dual semigroups. I. The sun-reflexive case. Math. Ann., 277 (1987), 709-725. III. Nonlinear Lipschitz continuous perturbations in the sun-reflexive case. "Volterra Integro Differential Equations in Banach Spaces and Applications", Trento 1987. (eds. G. Da Prato, M. Iannelli), Longman, Harlow, Essex, 1989, 95-116.

[3] Ph. Clément, H.J.A.M. Heijmans et al., One-Parameter Semigroups. CWI Monographs 5, North-Holand, Amsterdam, 1987.

[4] K. Deimling, Ordinary Differential Equations in Banach Spaces. Lecture Notes in Math. 595, Springer, Berlin, 1977.

[5] O. Diekmann, H.J.A.M. Heijmans and H.R. Thieme, On the stability of the cell size distribution. J. Math. Biol., 19 (1984), 227-248.

[6] O. Diekmann, H.A. Lauwerier, T. Aldenberg and J.A.J. Metz, Growth, fission, and the stable size distribution. J. Math. Biol., 18 (1983), 135-148.

[7] G. Greiner and R. Nagel, Growth of cell populations via one-parameter semigroups of positive operators. Mathematics Applied to Science: In Memorian Edward D. Conway. (eds. J.A. Goldstein, S. Rosencrans and G.A. Sod) Proceedings Tulane University 1987, Academic Press, Inc., London, 1988, 79-105.

[8] H.J.A.M. Heijmans, On the stable size distribution of populations reproducing by fission into two unequal parts. Math. Biosci. 72 (1984), 19-50.

[9] T. Kato, Quasi-linear equations of evolution with applications to partial differential equations. Spectral Theory and Differential Equations (ed. W.N. Everitt), Proceedings Dundee 1974, Lecture Notes in Math. 448, Springer, Berlin, 1975, 25-70.

[10] S.A.L.M. Kooijman and J.A.J. Metz, On the dynamics of chemically stressed populations: the deduction of population consequences from effects on individuals. Ecotox. Env. Saf., 8 (1984), 254-274.

[11] R.H. Martin, Nonlinear Operators and Differential Equations in Banach Spaces. John Wiley and Sons, New York, 1976.

[12] J.A.J. Metz and O. Diekmann (eds.), Dynamics of Physiologically Structured Populations. Lecture Notes in Biomath. 86, Springer, Berlin, 1986.

[13] L.F. Murphy, A nonlinear growth mechanism in size structured population dynamics. J. Theor. Biol., 104 (1983), 493-506. 
[14] R. Nagel (ed.), One-Parameter Semigroups of Positive Operators. Lecture Notes in Math., 1184, Springer, Berlin, 1986.

[15] A. Pazy, Semigroups of Linear Operators and Applications to Partial Differenial Equations. Springer, New York, 1983.

[16] H.H. Schaefer, Banach Lattices and Positive Operators. Springer, Berlin, 1974.

[17] S.L. Tucker and S.O. Zimmerman, A nonlinear model of population dynamics containing an arbitrary number of continuous structure variables. SIAM J. Appl. Math., 48 (1988), 549-591. 